\title{
Size at birth, lifecourse factors, and cognitive function in late life: findings from the MYsore study of Natal effects on Ageing and Health (MYNAH) cohort in South India
}

\author{
Murali Krishna, ${ }^{1,2}$ (D) Ghattu V. Krishnaveni, ${ }^{1}$ Veena Sargur, ${ }^{1}$ Kalyanaraman Kumaran, ${ }^{1,3}$ \\ Mohan Kumar, Kiran Nagaraj, ${ }^{1}$ Patsy Coakley, ${ }^{4}$ Samuel Chirstaprasad Karat, \\ Giriraj R. Chandak, ${ }^{5}$ Mathew Varghese, ${ }^{6}$ Martin Prince, ${ }^{7}$ Clive Osmond, ${ }^{3}$ \\ and Caroline H.D. Fall ${ }^{3}$ \\ ${ }^{1}$ CSI Holdsworth Memorial Hospital, Mandimohalla, Mysore, India \\ ${ }^{2}$ Foundation for Research and Advocacy in Mental Health Mysore, Mysore, India \\ ${ }^{3}$ MRC Lifecourse Epidemiology Unit, University of Southampton, Southampton, UK \\ ${ }^{4}$ Lifecourse Epidemiology Unit, University of Southampton, Southampton, UK \\ ${ }^{5}$ CSIR-Centre for Cellular and Molecular Biology, Hyderabad, India \\ ${ }^{6}$ National Institute of Mental Health and Neurosciences, Bangalore, India \\ ${ }^{7}$ Institute of Psychiatry, Kings College London, London, UK
}

Objective: To examine if smaller size at birth, an indicator of growth restriction in utero, is associated with lower cognition in late life, and whether this may be mediated by impaired early life brain development and/or adverse cardiometabolic programming.

Design: Longitudinal follow-up of a birth cohort.

Setting: CSI Holdsworth Memorial Hospital (HMH), Mysore South India.

Participants: 721 men and women (55-80 years) whose size at birth was recorded at HMH. Approximately 20 years earlier, a subset $(n=522)$ of them had assessments for cardiometabolic disorders in mid-life.

Measurements: Standardized measurement of cognitive function, depression, sociodemographic, and lifestyle factors; blood tests and assessments for cardiometabolic disorders

Results: Participants who were heavier at birth had higher composite cognitive scores (0.12 SD per SD birth weight $[95 \%$ CI $0.05,0.19] p=0.001)$ in late life. Other lifecourse factors independently positively related to cognition were maternal educational level and participants' own educational level, adult leg length, body mass index, and socioeconomic position, and negatively were diabetes in mid-life and current depression and stroke. The association of birth weight with cognition was independent cardiometabolic risk factors and was attenuated after adjustment for all lifecourse factors $(0.08$ SD per SD birth weight [95\% CI - 0.01, 0.18] $p=0.07)$.

Conclusions: The findings are consistent with positive effects of early life environmental factors (better fetal growth, education, and childhood socioeconomic status) on brain development resulting in greater long-term cognitive function. The results do not support a pathway linking poorer fetal development with reduced late life cognitive function through cardiometabolic programming.

Key words: birth weight, cognition, cardiometabolic disorders, Developmental Origins of Health and Disease (DOHaD), Low- and Middle-Income Countries (LMIC)

Correspondence should be addressed to: Caroline H.D. Fall, Lifecourse Epidemiology Unit, University of Southampton, University Road, Southampton SO17 1BJ, UK. Phone: +44(0)23 8059 5000. Email: chdf@ mrc.soton.ac.uk Received 26 Oct 2020; revision requested 11 Jan 2021; revised version received 22 Aug 2021; accepted 23 Aug 2021. First published online 20 October 2021.

\section{Introduction}

Neurocognitive disorders are a major cause of disability and mortality in late life and are associated with high costs for health systems and society (Lim et al., 2012; World Alzheimer Report, 2014). For late life neurocognitive disorders, as for other late life chronic 
diseases, there is an increased interest in the concept that environmental factors in early life (fetal life and childhood) may have an impact on the risk of disease (Developmental Origins of Health and Disease or "DOHaD" hypothesis). There are two plausible pathways linking early experience to cognitive aging: (a) by a direct effect of reduced intrauterine nutrition (reflected in birth size) on fetal brain development leading to reduced cognitive reserve and decreased cognitive ability, and (b) programming of metabolism in very early life by under-nutrition leading to increased risk mediated through cardiometabolic disorders (Whalley et al., 2006).

Quality of nutrition during intrauterine development, reflected crudely in size at birth, is an important determinant of lifelong function, health, and disease risk (Barker et al., 1993). Birth weight and head circumference at birth are indicators of intrauterine growth and brain development, respectively (Epstein and Epstein, 1978; Gunnell, 2002). Larger birth weight, the most widely researched birth size measure, is associated with better cognitive function and higher intelligence from infancy through the third decade of life in several populations and countries independent of social background (Krishna et al., 2019; Shenkin et al., 2004; Grove et al., 2017). This association of birth weight with cognition occurs across the whole spectrum of birth weight rather than being confined to an extreme group. However, the strength of this association is known to diminish as individuals reach middle age and may not persist beyond mid-life (Grove et al., 2017). The DOHaD pathways to late life cognition have not been systematically explored in previous birth cohort studies, most of which are from higherincome settings. Therefore, we examined the relationship between size at birth and cognitive function in late life and explored the pathways of $\mathrm{DOHaD}$ cognitive aging in the MYNAH (MYsore studies of Natal effect on Ageing and Health) cohort, in Mysore, South India.

This study examined if smaller size at birth was associated with poorer scores in tests of cognitive function in 721 men and women aged 55-80 years from the matched birth records cohort at Holdsworth Memorial Hospital (HMH) in Mysore, South India. The mediating effects of childhood growth, education, and cardiometabolic risk factors and socioeconomic position in adult life were examined in order to explore the two possible plausible nutrition-related pathways linking prenatal growth to cognitive function in late life: the $\mathrm{DOHaD}$ cognitive reserve and or the programmed cardiometabolic pathways of cognitive aging (Figure 1).

\section{Materials and methods}

\section{Setting}

This study was carried out at the Epidemiology Research Unit (ERU), HMH in Mysore, South India. The study was approved by the Hospital Research and Ethics Committee. HMH has preserved obstetric records since 1934 till the present date. These include the birth weight, length, and head circumference of all babies born in the hospital. During 1993-2001, the records were used to trace people born in the hospital between 1934 and 1966. The tracing process resulted in 3,427 men and women being matched to their birth records, and 1069 (mean 46.7 years SD [4.7]) of them were examined (1993-1995) for associations of birth size with cardiometabolic risk factors in mid-life (Stein et al., 1996), At the time of the first study, information about parental education and occupation at the time of participants' birth was obtained.

The cohort profile and findings from previous studies have been published elsewhere (Krishna et al., 2015). The cohort members were re-traced between 2012-2015, and 721 (408 men and 313 women born before 1955 , i.e. above 55 years of age) were examined in this study (Figure 2).

\section{Assessments}

All participants underwent standardized assessments for sociodemographic characteristics, cardiometabolic risk factors, cognitive function, and mental health, details of which have been published in a study protocol (Krishna et al., 2017) and are briefly summarized here:

(a) Cognitive assessments: The 10/66 Dementia Research Battery was administered in local languages by a trained clinical psychologist (Prince et al., 2007). This included the following: (i) Community Screening Instrument for Dementia (CSID), a 32-item test assessing orientation, comprehension, memory, naming, and language (Hall et al., 2000), (ii) animal naming task (Hall et al., 2000; Morris et al., 1989) from the CERAD (Centre for European Registry for Alzheimer's disease) test for assessing verbal fluency. A score of one point was given for each valid name generated in a space of a minute, (iii) Word List Memory and Recall test (WLMR) adapted from the CERAD and used in the Indo-USA Ballabgarh study to evaluate immediate and delayed recall, respectively (Ganguli et al., 1996). In the learning phase, a list of 10 words was read out to the participant who was then asked to recall as many as possible immediately. This process was repeated three times, giving the subject a score out 


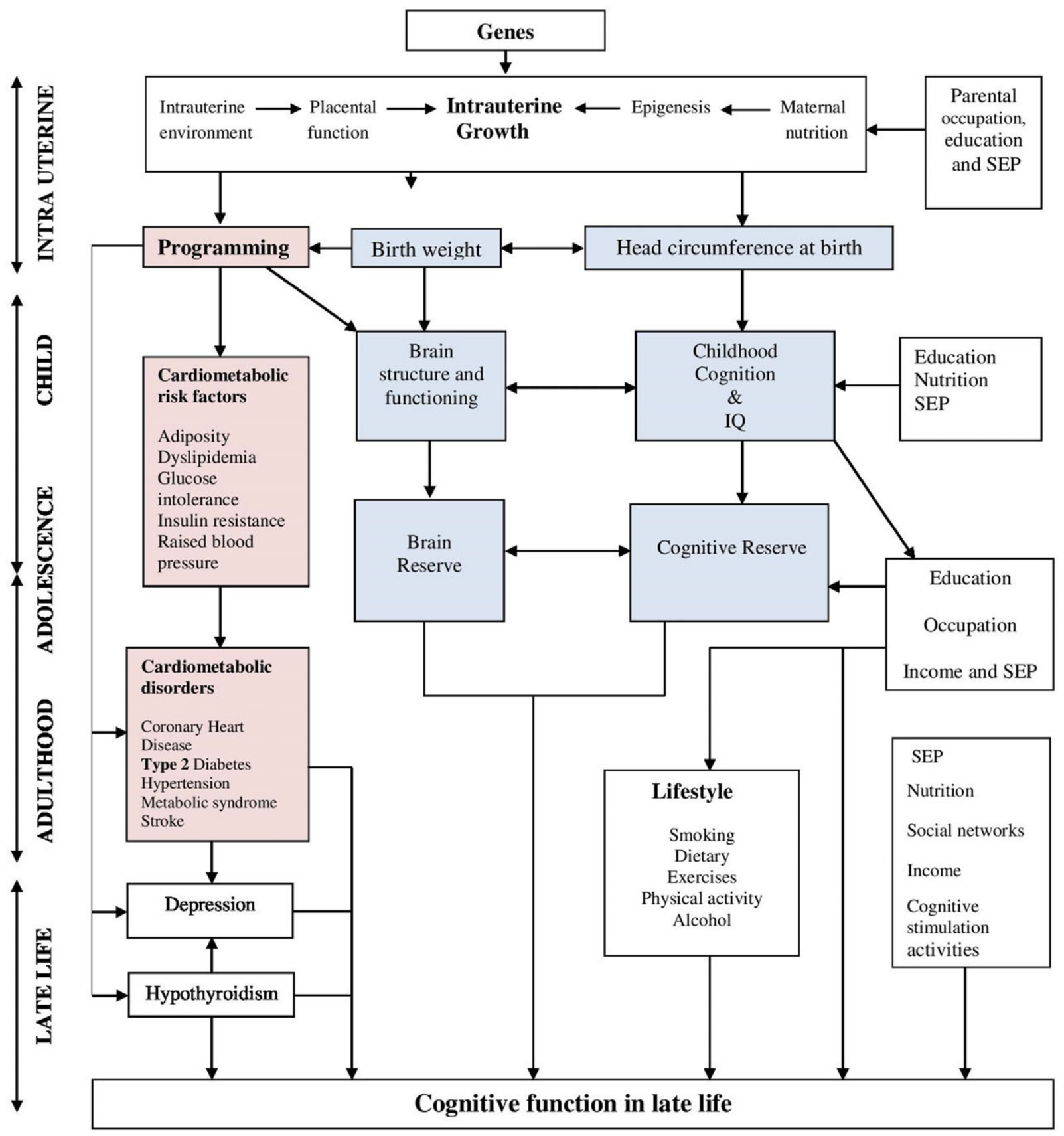

Figure 1. Illustration of the $\mathrm{DOHaD}$ cardiometabolic (in pink) and cognitive reserve (in blue) pathways to cognitive aging.

of 30. Approximately five minutes later, the participant was again asked to recall the 10 words, giving a recall score out of 10 and total score of 40 , and (iv) the CSID informant interview with a reliable informant for evidence of cognitive and functional decline. The frequency of six specific and characteristic memory lapses (forgetting where s/he has put things, where things are kept, names of friends, names of family, when s/ he last saw informant, and what happened the day before) was obtained. If the subject was receiving care, the primary caregiver was considered a reliable informant. (Hendrie et al., 1993; Prince et al., 2003).

(b) Geriatric Mental State (GMS) Examination, a standardized structured interview for diagnosis of depression and other mental disorders, was administered by a trained psychiatrist (MK) (Copeland et al., 1986).

(c) Physical health assessments included pulse rate, systolic and diastolic resting blood pressure, weight, height, leg length, head circumference, waist and hip circumference, and skinfold thickness according to the $10 / 66$ protocol 


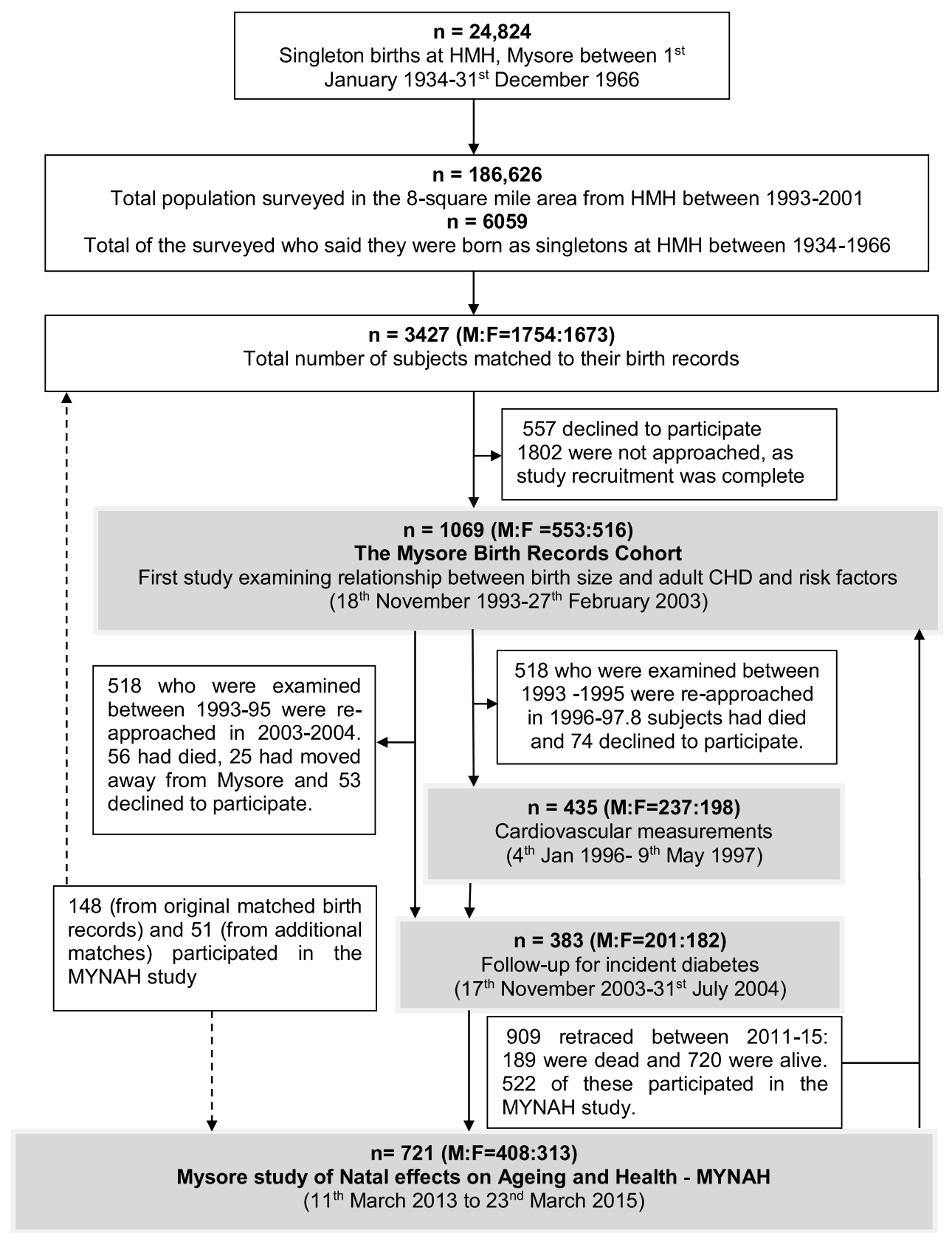

Figure 2. The Mysore Birth Records Cohort studies.

(Prince et al., 2007), 12-lead ECG for Minnesota coding (Macfarlane, 2000), structured neurological examination, and Rose Angina Questionnaire (Cook et al., 1989)

(d) A structured interview for specific cognitive risk factors, lifestyle, and cardiovascular risk factors (Prince et al., 2007), and Standard of Living Index questionnaire to estimate socioeconomic status. (National Family Health Survey, 2006)

(e) Blood tests: Oral glucose tolerance test, fasting insulin, and lipid profile (total cholesterol, highdensity lipoprotein, low-density lipoprotein, and triglycerides).

\section{Definitions}

(a) Dementia was defined as a score above a cutoff point of predicted probability of DSM IV Dementia Syndrome from the logistic regression equation of the 10/66 dementia diagnostic algorithm (American Psychiatric Association, 2000; Prince et al., 2003).

(b) Diagnosis of depression was derived from the GMS Examination using its computerized algorithm (GMS) (Copeland et al., 1986).

(c) Diabetes mellitus was diagnosed if fasting plasma glucose was $\geq 7$ or 2 -hour plasma glucose was $\geq 11.1 \mathrm{mmol} / \mathrm{l}$, or if the participant was known to 
have diabetes and was taking medication for diabetes (WHO, 2006).

(d) Insulin resistance was estimated using the Homeostasis Model Assessment equation for insulin resistance (HOMA-IR) (Matthews et al., 1985).

(e) Hypertension was defined as a systolic blood pressure $\geq 130 \mathrm{mmHg}$ or diastolic blood pressure $\geq$ $85 \mathrm{mmHg}$ or (International Diabetes Federation criteria Alberti et al., 2006) a diagnosis of hypertension by a doctor, for which they were taking antihypertensive medication.

(f) Metabolic syndrome was defined using International Diabetes Federation criteria (Alberti et al., 2006) for South Asians as central obesity (waist circumference $\geq 90 \mathrm{~cm}$ for men and $\geq 80 \mathrm{~cm}$ for women) plus any two of the following four factors: raised triglyceride level ( $\geq 1.7 \mathrm{mmol} / \mathrm{l})$, low HDL cholesterol $(<1.03 \mathrm{mmol} / 1$ in males and $<1.29$ $\mathrm{mmol} / \mathrm{l}$ in females), raised blood pressure (systolic pressure $\geq 130$ or diastolic pressure $\geq 85 \mathrm{mmHg}$, or on treatment for previously diagnosed hypertension) or raised fasting plasma glucose $>5.6$ $\mathrm{mmol} / \mathrm{l}$, or previously diagnosed type 2 diabetes.

(g) Coronary heart disease (CHD) was diagnosed if there was typical angina on the Rose chest pain questionnaire or a history of cardiac revascularization procedures or the presence of Minnesota codes 1-1 or 1-2 (major Q waves) on the ECG.

(h) Stroke was diagnosed based on the 10/66 protocol: self-report ("have you ever been told by a doctor that you had a stroke?"), history of symptoms, and findings from a structured neurological examination (Prince et al., 2007).

\section{Indicators of child environment}

As study participants were not examined during their childhood, we have considered the following as proxy indicators of the childhood milieu: maternal educational level at the time of the participants' birth for childhood environment, adult leg length for childhood growth, attained educational level for cognitive reserve, and adult head circumference for brain development in childhood (i.e. brain reserve) (Sudfield et al., 2015).

\section{Data analyses}

We carried out representativeness analyses to examine differences in size at birth between the originally matched 3427 men and women with: all the births at HMH between 1934 and 1966, the 1069 who participated in the baseline study, and the 721 who were examined in this study. Subgroup analyses between those who were successfully retraced and lost to follow-up and between those who were alive and dead when retraced for this study were also conducted (Supplementary Tables S1-S3).
We examined the measurements of size at birth and current sociodemographic, anthropometric, cardiometabolic, cognitive, and mental health characteristics of the study participants, stratified by sex. Differences in means and medians between the groups were examined using $t$-tests and MannWhitney $U$-tests, respectively. Differences in proportions between the groups were examined using chi-square tests.

Principal component analysis was employed to derive a composite cognitive score from the 4 cognitive domains from the 10/66 tests.

Cognitive function was measured for the first time in this study, and such data in mid-life were not available for the study participants. In addition, pathway variables related to cardiometabolic health in mid-life were only available in a subset of the participants. Different instruments were used to measure socioeconomic position in mid-life and late life, with different constructs. Due to the above factors, we were unable to employ Structural Equational Modeling to construct the pathways though it was best suited to handle the unobserved variables like cognitive reserve.

The 721 study participants were the offspring of 421 marriages, and siblings were more similar in their standardized cognitive function scores than those who were unrelated. The between-sibship variance of 0.203 was 3.5 times larger than its standard error $(0.058, p=0.0005)$, and the intraclass correlation was 0.20 . So, to account for the influence of sibship, mixed linear regression analyses, adjusting for sibship, were conducted to examine the associations of size at birth with continuous measures of cognitive function and mixed logistic regression analyses for binary outcomes. In the regression analyses, the log transform could make the data more normally distributed. These models were separately adjusted for cardiometabolic risk markers in mid-life and late life for evidence of DOHaD cardiometabolic pathway. They were adjusted for indicators of brain reserve (head circumference) and cognitive reserve (attained educational levels) for evidence of the $\mathrm{DOHaD}$ cognitive reserve pathway. If the associations of size at birth with cognition were mediated or moderated by cardiometabolic risk factors or by indicators of cognitive reserve, this will result in noticeable attenuation in the effect sizes ( $\beta$ standardized regression coefficients) of corresponding models. Finally, multivariate mixed models were constructed to examine the confounding or mediating effect of factors across the lifecourse on the relationship between measurements of size at birth and cognitive function in late life. All analyses were conducted using SPSS version 
Table 1. Characteristics of study participants by sex

\begin{tabular}{|c|c|c|c|c|c|}
\hline & \multicolumn{2}{|c|}{ MEN $n=408$} & \multicolumn{2}{|c|}{ WOMEN $n=313$} & $P$ \\
\hline \multicolumn{6}{|l|}{ Demographic characteristics } \\
\hline Age $(\text { years })^{a}$ & 62.0 & $(5.2)$ & 62.8 & $(5.4)$ & 0.25 \\
\hline \multicolumn{6}{|l|}{ Marital status $n(\%)$} \\
\hline Never married & 12 & (3) & 11 & (3) & \\
\hline Married & 370 & $(90)$ & 189 & $(60)$ & 0.30 \\
\hline Widowed & 17 & $(4)$ & 106 & (34) & \\
\hline Divorced/separated & 9 & (3) & 7 & (3) & \\
\hline \multicolumn{6}{|l|}{ Education $n(\%)$} \\
\hline Illiterate & 7 & $(2)$ & 15 & $(5)$ & \\
\hline Primary & 49 & $(12)$ & 46 & $(15)$ & \\
\hline Secondary & 105 & $(26)$ & 87 & $(28)$ & 0.18 \\
\hline Pre-university & 78 & (19) & 67 & $(21)$ & \\
\hline Diploma & 53 & (13) & 43 & $(14)$ & \\
\hline Graduate & 65 & $(16)$ & 18 & $(6)$ & \\
\hline Postgraduate & 51 & $(12)$ & 37 & (11) & \\
\hline SLI score ${ }^{\mathrm{a}}$ & 37.3 & 7.4 & 36.4 & 8.0 & 0.10 \\
\hline \multicolumn{6}{|l|}{ Current occupation $n(\%)$} \\
\hline \multicolumn{6}{|l|}{ Not in paid employment } \\
\hline Unemployment & 8 & $(2)$ & 3 & $(1)$ & \\
\hline Homemaker & 5 & $(1)$ & 207 & $(66)$ & 0.64 \\
\hline Retired & 146 & $(36)$ & 53 & $(17)$ & \\
\hline In paid employment & 249 & $(61)$ & 50 & $(16)$ & \\
\hline \multicolumn{6}{|c|}{ Cardiometabolic and mental health characteristics } \\
\hline $\mathrm{BMI}\left(\mathrm{kg} / \mathrm{m}^{2}\right)^{\mathrm{a}}$ & 25.2 & $(4.6)$ & 28.4 & $(5.5)$ & $<0.001$ \\
\hline Underweight $n(\%)$ & 28 & (7) & 8 & (3) & \\
\hline Normal $n(\%)$ & 184 & $(45)$ & 76 & $(24)$ & $<0.001$ \\
\hline Overweight $n(\%)$ & 136 & (33) & 120 & $(39)$ & \\
\hline Obese $n(\%)$ & 59 & $(15)$ & 106 & $(34)$ & \\
\hline Head circumference $(\mathrm{cm})^{\mathrm{a}}$ & 53.1 & (1.1) & 52.3 & (1.4) & $<0.001$ \\
\hline Leg length $(\mathrm{cm})^{\mathrm{a}}$ & 93.7 & $(14.9)$ & 85.1 & $(4.5)$ & $<0.001$ \\
\hline Diabetes $n(\%)$ & 192 & $(47)$ & 149 & $(48)$ & 0.79 \\
\hline Hypertension $n(\%)$ & 231 & $(57)$ & 214 & $(69)$ & 0.001 \\
\hline Metabolic syndrome $n(\%)$ & 243 & $(60)$ & 215 & $(60)$ & 0.01 \\
\hline Coronary heart disease $n(\%)$ & 104 & $(25)$ & 95 & (30) & 0.15 \\
\hline Stroke $n(\%)$ & 19 & $(5)$ & 2 & $(1)$ & $<0.001$ \\
\hline Depression $n(\%)$ & 54 & (13) & 84 & $(27)$ & $<0.001$ \\
\hline Dementia $n(\%)$ & 12 & (3) & 10 & (3) & 0.84 \\
\hline \multicolumn{6}{|l|}{ Size at birth } \\
\hline Birth weight $(\mathrm{kg})^{\mathrm{a}}$ & 2.8 & $(0.4)$ & 2.7 & $(0.4)$ & 0.003 \\
\hline Head circumference $(\mathrm{cm})^{\mathrm{a}}$ & 33.7 & $(1.7)$ & 33.3 & $(1.5)$ & 0.005 \\
\hline Length at birth $(\mathrm{cm})^{\mathrm{a}}$ & 48.2 & $(2.1)$ & 47.7 & $(2.9)$ & 0.03 \\
\hline Ponderal index $\left(\mathrm{kg} / \mathrm{m}^{3}\right)^{\mathrm{a}}$ & 25.4 & $(4.6)$ & 25.4 & $(5.1)$ & 0.88 \\
\hline
\end{tabular}

Difference between the groups was examined using $t$-test for means. Differences with categorical variables were examined using $\chi^{2}$ tests. SLI: standard of living index.

${ }^{\mathrm{a}}$ Mean (SD).

23.0 (IBM SPSS Statistics for Macintosh, Version 23.0).

\section{Results}

Sociodemographic, physical, and mental health characteristics, and measurements of size at birth of the study participants are provided in Table 1.
Weight, length, and head circumference at birth were higher in men than women, but ponderal index at birth was similar. Just over a quarter of the participants weighed less than $2.5 \mathrm{~kg}$ at birth (low birth weight), only $5 \%$ weighed $3.5 \mathrm{~kg}$ and above.

Compared to men, women scored higher on word recall tests $(\beta=1.52$ [95\% CI $0.93,2.10])$ for immediate recall (score) and ( $\beta=0.80[95 \%$ CI $0.50,1.09]$ ) for delayed recall (score); 
$p<0.001$ for both. The composite cognitive score decreased with age and increased with education, socioeconomic status, and occupational levels $(\beta=-0.03 \mathrm{SD}$ per year of age $[95 \% \mathrm{CI}$ $-0.04,-0.02])$; $(\beta=0.29$ SD per level of education [95\% CI $0.25,0.33]) ;(\beta=0.04$ SD per unit of SLI [95\% CI $0.03,0.05])$ and $(\beta=0.31$ SD per level of occupation [95\% CI 0.23, 0.39]; $p<0.001$ for all).

Birth weight was directly associated with scores across all cognitive function tests and composite cognitive score, while length at birth was directly associated with scores on the delayed recall test and composite cognitive score (Table 2). For example, a $418 \mathrm{~g}$ (1 SD) higher birth weight or a $3 \mathrm{~cm}$ (1 SD) longer length at birth was associated with a $0.1 \mathrm{SD}$ higher composite cognitive score in late life. There were no significant interactions between sex and birth weight for cognitive outcomes indicating that associations of birth weight with cognitive outcomes were similar for men and women.

\section{The DOHaD cardiometabolic programming pathway of cognitive aging}

A subset of the $721 \quad(n=522: 299$ men and 223 women) were examined 20 years earlier when they were aged 40-67 years ("mid-life": mean 46.7 years SD [4.7]) for cardiometabolic risk factors using same standardized physical health assessments and blood tests, and definitions for diagnosis as in the current study.

Higher birth weight was associated with higher mid-life and late life BMI, waist circumference and skinfolds, and higher mid-life insulin resistance and mid-life metabolic syndrome (Supplementary Tables S4 and S5). After adjusting for adult BMI, birth weight was unrelated to cardiometabolic risk factors in mid-life and late life.

Men and women with greater BMI, waist circumference, and skinfolds in mid-life and late life had higher composite cognitive scores in late life (Supplementary Table S6). Participants with diabetes in mid-life had lower composite cognitive scores in late life, while those with higher levels of total cholesterol and LDL cholesterol in mid-life had higher composite cognitive scores in late life.

Fasting insulin concentrations in late life were directly related to composite cognitive score in late life, while diastolic blood pressure and stroke in late life were inversely associated with composite cognitive score in late life.

In mixed effects models (Supplementary Table S7), the direct association of birth weight with composite cognitive score was not attenuated after separately adjusting for cardiometabolic risk factors in mid-life and late life, indicating that the birth weight-cognition relationship was independent of and not mediated by cardiometabolic risk factors in mid-life and late life. These findings were therefore not consistent with the $\mathrm{DOHaD}$ programmed cardiometabolic pathway of cognitive aging in the MYNAH cohort.

\section{The DOHaD cognitive reserve pathway of cognitive aging}

Men and women with larger size at birth had larger head circumference $(\mathrm{cm})$ in late life (a proxy for brain reserve) $(\beta=0.8 \mathrm{~cm}$ per $\mathrm{kg}[95 \%$ CI $0.62,1.12] p=$ $<0.001)$ for birth weight; $(\beta=0.03 \mathrm{~cm}$ per $\mathrm{cm}[95 \%$ CI $-0.00,0.07] p=0.07)$ for birth length; $(\beta=0.08$ $\mathrm{cm}$ per $\mathrm{cm}$ [95\% CI 0.01, 0.15] $p=0.02)$ for head circumference at birth; $\beta=0.03 \mathrm{~cm}$ per $\mathrm{kg} / \mathrm{m}^{3}[95 \%$ CI $0.01,0.05] p=0.01$ for ponderal index). Birth weight, but not other measurements of birth size, was directly related to attained educational levels (a proxy for cognitive reserve) $(\beta=0.29$ levels of education per $\operatorname{kg}[95 \%$ CI $0.01,0.56] p=0.04)$. Adult head circumference and attained educational level were positively correlated $(r=0.2,[p<0.001])$. Further, men and women with larger head circumference as adults (brain reserve) and higher attained educational level (cognitive reserve) had higher composite cognitive scores in late life for current head circumference $\left(\beta=0.06\right.$ SD per cm $\left[\begin{array}{lllll}95 \% & \text { CI } & 0.01, & 0.10\end{array}\right]$ $p=0.02)$; for education $(\beta=0.30 \mathrm{SD}$ per level of education [95\% CI $0.26,0.33] p<0.001)$. There were no significant interactions between birth weight and education, or birth weight and adult head circumference for cognitive outcomes.

In a mixed effects regression model, the association of birth weight with composite cognitive score was marginally attenuated by adjusting for indicators of brain reserve or cognitive reserve separately or together, but the association remained significant $(\beta=0.20$ SD per kg $[95 \%$ CI $0.05,0.36] p=0.009)$ adjusted for adult head circumference and attained educational level, compared with $(\beta=0.29$ [95\% CI $0.12,0.46], p=0.001$ ) (Table 2). This analysis indicated birth weight-cognition relationship may have been in part mediated by brain reserve and cognitive reserve, thus providing partial support for the cognitive reserve hypothesis of cognitive aging in this cohort.

Better environment and growth in childhood (indicated by maternal educational level at the time of birth and adult leg length) were directly related to higher composite cognitive score in this cohort $(\beta=0.25 \mathrm{SD}$ per level of maternal education [95\% CI $0.19,0.30] p<0.001)$ and $(\beta=0.04 \mathrm{SD}$ per $\mathrm{cm}$ of adult leg length [95\% CI 0.02, 0.05] $p<0.001)$. These associations were partly attenuated in mixed regression models adjusted for adult 
Table 2. Associations of size at birth with cognitive outcomes in late life

\begin{tabular}{|c|c|c|c|c|c|}
\hline $\begin{array}{l}\text { PREDICTOR } \\
\text { BIRTH SIZE }\end{array}$ & $\begin{array}{c}\text { GLOBAL COGNITION (SD) } \\
\beta(95 \% \mathrm{CI})^{\mathrm{a}} P\end{array}$ & $\begin{array}{c}\text { VERBAL FLUENCY (SCORE) } \\
\beta(95 \% \mathrm{CI})^{\mathrm{a}} P\end{array}$ & $\begin{array}{c}\text { IMMEDIATE RECALL } \\
\left(\text { SCORE) } \beta(95 \% \text { CI })^{\mathrm{a}} P\right.\end{array}$ & $\begin{array}{c}\text { DELAYED RECALL (SCORE) } \\
\beta(95 \% \mathrm{CI})^{\mathrm{a}} P\end{array}$ & $\begin{array}{c}\text { COMPOSITE COGNITIVE SCORE } \\
(\mathrm{SD})^{\mathrm{a}} \beta(95 \% \text { CI) } P\end{array}$ \\
\hline Birth weight (kg) & $0.28(0.11,0.46) 0.002$ & $0.95(0.24,1.60) 0.008$ & $0.82(0.14,1.50) 0.02$ & $0.37(0.58,1.15) 0.03$ & $0.29(0.12,0.46) 0.001$ \\
\hline $\begin{array}{l}\text { Birth weight } \\
\text { (SD) }\end{array}$ & $0.12(0.04,0.19) 0.002$ & $0.40(0.10,0.69) 0.008$ & $0.34(0.06,0.63) 0.02$ & $0.16(0.01,0.11) 0.03$ & $0.12(0.05,0.19) 0.001$ \\
\hline $\begin{array}{l}\text { Length at birth } \\
(\mathrm{cm})\end{array}$ & $0.02(0.00,0.04) 0.11$ & $0.05(-0.05,0.15) 0.34$ & $0.07(-0.03,0.17) 0.18$ & $0.06(0.01,0.11) 0.03$ & $0.03(0.00,0.05) 0.04$ \\
\hline $\begin{array}{l}\text { Length at birth } \\
\text { (SD) }\end{array}$ & $0.06(-0.01,0.13) 0.11$ & $0.15(-0.15,0.44) 0.34$ & $0.20(-0.09,0.50) 0.18$ & $0.17(0.02,0.32) 0.03$ & $0.07(0.00,0.15) 0.04$ \\
\hline $\begin{array}{l}\text { Head circumfer- } \\
\text { ence }(\mathrm{cm})\end{array}$ & $0.01(-0.04,0.06) 0.78$ & $0.05(-0.14,0.25) 0.60$ & $0.09(-0.10,0.28) 0.34$ & $0.05(-0.04,0.15) 0.26$ & $0.02(-0.02,0.07) 0.34$ \\
\hline $\begin{array}{l}\text { Head circumfer- } \\
\text { ence (SD) }\end{array}$ & $0.01(-0.06,0.09) 0.78$ & $0.08(-0.22,0.39) 0.60$ & $0.14(-0.15,0.45) 0.34$ & $0.09(-0.06,0.24) 0.26$ & $0.04(-0.04,0.11) 0.34$ \\
\hline $\begin{array}{l}\text { Ponderal index } \\
\qquad\left(\mathrm{kg} / \mathrm{m}^{3}\right)\end{array}$ & $0.00(-0.01,0.01) 0.96$ & $0.01(-0.05,0.06) 0.83$ & $-0.01(-0.07,0.06) 0.86$ & $-0.01(-0.04,0.02) 0.37$ & $-0.01(-0.09,0.06) 0.70$ \\
\hline $\begin{array}{l}\text { Ponderal index } \\
\text { (SD) }\end{array}$ & $0.00(-0.07,0.07) 0.96$ & $0.03(-0.27,0.33) 0.83$ & $-0.03(-0.33,0.27) 0.86$ & $-0.07(-0.22,0.08) 0.37$ & $-0.01(-0.09,0.06) 0.70$ \\
\hline
\end{tabular}

${ }^{a}$ Mixed linear regression analyses adjusted for age, sex, and sibship in which all exposures were treated as continuous variables.

Upon standardization, the expsoures carry SD as unit of measurement. 
head circumference and the participants' own attained education level $(\beta=0.08$ SD per level of maternal education at birth [95\% CI $0.03,0.14$ ] $p=0.003) ;(\beta=0.02 \mathrm{SD}$ per $\mathrm{cm}$ of leg length $[95 \%$ CI $0.01,0.03] p=0.005)$. This indicated that the direct associations of growth and environment in childhood with cognitive function in late life may be in part mediated by cognitive reserve.

\section{A lifecourse approach to cognitive aging in the MYNAH cohort}

In a series of lifecourse models (Table 3), including birth, childhood, and adult factors, there was attenuation of the association of birth weight with cognitive function. However, the borderline significant $p$ values for birth weight right across the table suggest a possible small but consistent effect of birth weight on cognitive function independent of attained education and SEP in mid-life, cardiometabolic factors, stroke, and depression in late life, and nutrition and lifestyle factors across the lifespan. Participants who were younger and women (compared to men) had higher composite cognitive scores in late life, independent of growth and environment in childhood, attained educational level and SEP in mid-life, cardiometabolic factors, stroke, and depression in late life, and nutrition and lifestyle factors across the lifespan.

In general, the effect size of the association of the participants' education level with their cognition in late life remained unchanged despite adjusting for factors across the lifecourse. In contrast, the effect size of the association of maternal education at the time of birth of the participants with offspring cognition in late life reduced by two-thirds when adjusted for the offspring's growth in early life and childhood, and their attained levels of education and SEP in adult life (model three), with no further reduction after additional adjustment for late life factors (model four). The direct effect of SEP on cognition in late life was attenuated but remained significant after adjusting for lifecourse factors. Smoking pack-years, hemoglobin, and levels of physical activity were inversely and significantly associated with cognition in late life in univariate analyses, but these were unrelated to cognition in mixed models after adjusting for all lifecourse factors.

This lifecourse model showed that childhood growth and environment (indicated by adult leg length and maternal education level, respectively), cognitive reserve (indicated by attained educational level), adult SEP, and a history of stroke were independently associated with cognitive function in late life in this cohort. There was some indication that prenatal growth (birth weight) had a small independent effect. The full set of predictors in this lifecourse model (model four) accounted for about $41 \%$ of variance in cognitive function in late life in this cohort (Table 3). After allowing for age and sex, the highest proportion of variance was attributable to attained education (29\%), followed by adult SEP (16\%), maternal educational level $(10 \%)$, childhood growth (10\%), and birth weight $(8 \%)$. These models remained unchanged when stratified by age (subgroup analysis) or by the size of sibship (sensitivity analysis).

\section{Discussion}

Birth weight and length, crude indicators of growth in fetal life, were positively associated with cognitive function in late life, possibly partially mediated by growth and environment in childhood, and cognitive reserve. Head circumference and ponderal index at birth were unrelated to cognitive function in late life. Growth and environment in childhood (as indicated by adult leg length and maternal education level), cognitive reserve (indicated by the participants' attained education level), and adult SEP were strongly positively and independently associated with cognitive function in late life in this cohort.

Findings from this study are consistent with some previous studies that have showed direct associations of birth weight with cognitive ability in childhood, adolescence, and young adulthood (Shenkin et al., 2004; Grove et al., 2017). The previous studies that have tested if body size at birth is associated with cognitive ability in late life have, however, produced contradictory findings (Grove et al., 2017; Krishna et al., 2019). Lower birth weight was associated with lower cognitive ability in three studies (Erickson et al., 2010; Costa et al., 2011; Raikkonen et al., 2013). In one other study, lower bi-parietal head diameter at birth, but not weight, length, ponderal index, head circumference, or occipitofrontal head diameter at birth, was associated with lower cognitive function in late life (Martyn et al., 1996). Birth weight and length at birth in one study (Shenkin et al., 2009), head diameter at birth in another (Gale et al., 2003), and head circumference and ponderal index at birth in a study from a low- and middleincome setting (China) (Zhang et al., 2009) were unrelated to cognitive abilities in late life.

The effect sizes of the associations of measurement of size at birth with various cognitive outcomes reported in the above studies have been synthesized in our systematic review (Krishna et al., 2017). Direct comparisons of all measurements of size at birth with all reported cognitive outcomes in our study with those from the above-mentioned studies 
Table 3. A mixed effects lifecourse model for cognitive aging in the MYNAH cohort

\begin{tabular}{|c|c|c|c|c|c|}
\hline \multicolumn{6}{|c|}{ COMPOSITE COGNITIVE SCORE (SD) } \\
\hline PREDICTOR & $\begin{array}{c}\text { UNADJUSTED } \\
\text { (UNIVARIATE ANALYSES) } \\
\beta(95 \% \mathrm{CI}) P\end{array}$ & $\begin{array}{l}\text { MODEL } 1 \beta \\
(95 \% \text { CI }) P\end{array}$ & $\begin{array}{l}\text { MODEL } 2 \beta \\
(95 \% \mathrm{CI}) P\end{array}$ & $\begin{array}{l}\text { MODEL } 3 \beta \\
(95 \% \mathrm{CI}) P\end{array}$ & $\begin{array}{l}\text { MODEL } 4 \beta \\
(95 \% \mathrm{CI}) P\end{array}$ \\
\hline Birth weight (SD) & $\begin{array}{l}0.1 \\
(0.03,0.18) \\
0.008\end{array}$ & $\begin{array}{l}0.12 \\
(0.05,0.19) \\
0.001\end{array}$ & $\begin{array}{l}0.06 \\
(-0.01,0.12) \\
0.11\end{array}$ & $\begin{array}{l}0.05 \\
(-0.01,0.12) \\
\quad 0.1\end{array}$ & $\begin{array}{l}0.08 \\
(-0.01,0.18) \\
\quad 0.07\end{array}$ \\
\hline Age (years) & $\begin{array}{l}-0.03 \\
(-0.04,-0.02) \\
\quad<0.001\end{array}$ & $\begin{array}{l}-0.04 \\
(-0.05,-0.03) \\
\quad 0.001\end{array}$ & $\begin{array}{l}-0.03 \\
(-0.04,-0.01) \\
\quad<0.001\end{array}$ & $\begin{array}{l}-0.03 \\
(-0.04,-0.02) \\
<0.001\end{array}$ & $\begin{array}{l}-0.02 \\
(-0.04,-0.00) \\
0.04\end{array}$ \\
\hline $\operatorname{Sex}(0=M, 1=F)$ & $\begin{array}{l}0.28 \\
(0.14,0.42) \\
<0.001\end{array}$ & $\begin{array}{l}0.33 \\
(0.19,0.47) \\
<0.001\end{array}$ & $\begin{array}{l}0.57 \\
(0.39,0.75) \\
<0.001\end{array}$ & $\begin{array}{l}0.54 \\
(0.38,0.71) \\
<0.001\end{array}$ & $\begin{array}{l}0.65 \\
(0.37,0.93) \\
<0.001\end{array}$ \\
\hline $\begin{array}{l}\text { Maternal education level } \\
\text { at the time of birth }\end{array}$ & $\begin{array}{l}0.26 \\
(0.20,0.32) \\
<0.001\end{array}$ & & $\begin{array}{l}0.23 \\
(0.17,0.28) \\
<0.001\end{array}$ & $\begin{array}{l}0.07 \\
(0.02,0.13) \\
0.008\end{array}$ & $\begin{array}{l}0.08 \\
(0.00,0.16) \\
0.04\end{array}$ \\
\hline Adult leg length $(\mathrm{cm})$ & $\begin{array}{l}\quad 0.01 \\
(-0.01,0.02) \\
0.36\end{array}$ & & $\begin{array}{l}0.03 \\
(0.01,0.04) \\
<0.001\end{array}$ & $\begin{array}{l}0.01 \\
(0.00,0.03) \\
0.03\end{array}$ & $\begin{array}{l}0.01 \\
(0.01,0.03) \\
0.01\end{array}$ \\
\hline Education $^{\mathrm{a}}$ & $\begin{array}{l}0.29 \\
(0.25,0.33) \\
<0.001\end{array}$ & & & $\begin{array}{l}0.22 \\
(0.17,0.27) \\
0.02\end{array}$ & $\begin{array}{l}0.21 \\
(0.15,0.28) \\
<0.001\end{array}$ \\
\hline SLI in late life (score) & $\begin{array}{l}0.04 \\
(0.03,0.05) \\
<0.001\end{array}$ & & & $\begin{array}{l}0.02 \\
(0.01,0.03) \\
<0.001\end{array}$ & $\begin{array}{l}0.01 \\
(0.01,0.02) \\
0.006\end{array}$ \\
\hline $\begin{array}{l}\text { Metabolic syndrome late } \\
\text { life }(0=\text { no, } 1=\text { yes })\end{array}$ & $\begin{array}{l}0.15 \\
(0.00,0.30) \\
0.04\end{array}$ & & & & $\begin{array}{l}-0.11 \\
(-0.30,0.08) \\
0.23\end{array}$ \\
\hline $\begin{array}{l}\text { Stroke in late life } \\
\qquad(0=\text { no, } 1=\text { yes })\end{array}$ & $\begin{array}{l}-0.92 \\
(-1.35,-0.50) \\
<0.001\end{array}$ & & & & $\begin{array}{l}-0.68 \\
(-1.21,0.16) \\
\quad 0.01\end{array}$ \\
\hline $\begin{array}{l}\text { Depression in late life } \\
(0=\text { no, } 1=\text { yes })\end{array}$ & $\begin{array}{l}-0.47 \\
(-0.65,-0.29) \\
<0.001\end{array}$ & & & & $\begin{array}{l}-0.04 \\
(-0.28,0.19) \\
\quad 0.69\end{array}$ \\
\hline $\begin{array}{l}\text { Haemoglobin in late life } \\
\quad(\mathrm{g} \%)\end{array}$ & $\begin{array}{l}\quad 0 \\
(-0.04,0.04) \\
\quad 0.96\end{array}$ & & & & $\begin{array}{l}0.06 \\
(0.01,0.09) \\
0.09\end{array}$ \\
\hline Smoking (pack-years) & $\begin{array}{l}-0.01 \\
(-0.01,0.00) \\
<0.001\end{array}$ & & & & $\begin{array}{l}\quad 0 \\
(-0.00,0.00) \\
0.26\end{array}$ \\
\hline $\begin{array}{l}\text { Alcohol in late life } \\
\text { (units/week) }\end{array}$ & $\begin{array}{l}0 \\
(-0.00,0.00) \\
0.59\end{array}$ & & & & $\begin{array}{l}\quad 0 \\
(-0.00,0.01) \\
0.35\end{array}$ \\
\hline $\begin{array}{l}\text { Physical activity in late } \\
\text { life }^{\mathrm{b}}\end{array}$ & $\begin{array}{l}-0.21 \\
(-0.37,-0.04) \\
0.01\end{array}$ & & & & $\begin{array}{l}-0.1 \\
(-0.31,0.10) \\
0.33\end{array}$ \\
\hline $\begin{array}{l}\text { Marital status in late life } \\
(0=\text { married } 1=\text { wi- } \\
\text { dowed })\end{array}$ & $\begin{array}{l}-0.25 \\
(-0.37,-0.13) \\
0.003\end{array}$ & & & & $\begin{array}{l}-0.17 \\
(-0.41,0.06) \\
\quad 0.15\end{array}$ \\
\hline $\begin{array}{l}\text { Estimate of the variance ex } \\
\text { each model }\end{array}$ & xplained by the predictors in & 0.08 & 0.19 & 0.33 & 0.41 \\
\hline
\end{tabular}

Model 1: Adjusted for factors from early life (birth weight), age, sex, and sibship.

Model 2: Adjusted for factory from early life (birth weight) and childhood (maternal education, adult leg length) age, sex, and sibship.

Model 3: Adjusted for factors from early life, childhood, and adult life (SLI and education), age, sex, and sibship.

Model 4: Adjusted for factors from early life, childhood, adult life, and late life factors (metabolic syndrome, stroke, depression, marital status), nutritional and life style factors across the life (haemoglobin, smoking, alcohol, and physical activity).

SLI: standard of living index.

${ }^{a}$ Education per level: $0=$ illiterate, $1=$ primary, $2=$ secondary, $3=$ pre-university, $4=$ college, $5=$ graduate, $6=$ postgraduate

${ }^{\mathrm{b}}$ Physical activity: $0=$ sedentary, $1=$ mild, $2=$ moderate, $3=$ strenuous. 
are complicated by differences in the populations examined, methodology, study design, and measurements of cognitive function. When feasible to compare, the effect sizes of birth weight with verbal fluency and delayed recall observed in our study are comparable to those reported in other birth cohort studies, for example, a kilogram increase in birth weight was related to an increase in verbal fluency by 0.7 words in Costa et al. (2011), 0.8 words in Erickson et al. (2010), 0.9 words in Shenkin et al. (2009), and 0.9 words in our study; delayed recall by 0.3 words in Costa et al. (2011), 0.8 words in Erickson et al. (2010), and 0.4 words in our study.

In this study, there was no evidence to support the $\mathrm{DOHaD}$ hypothesis that programming of cardiometabolic outcomes due to reduced prenatal growth and development had resulted in lower cognitive function in late life. None of the previously published twelve birth cohort studies (Krishna et al., 2019) were set up to explore the pathways of cognitive aging in a manner described in this study. However, similar to our study, the direct effects of birth size measures with late life cognition were independent of stroke and CHD (Raikkonen et al., 2013), diabetes and hypertension (Costa et al., 2011), and diabetes and CHD (Hyvarinen et al., 2009). In general, previous birth cohort studies are uninformative about the relationship between birth size and the above-mentioned cardiometabolic disorders and are limited to reporting the birth size-cognition associations. Furthermore, to the best of our knowledge, we have for the first time explored mid-life cardiometabolic factors as pathway variables.

The direct association of birth weight with cognitive function in late life was partly attenuated by adjusting for attained educational level of the participants, a proxy indicator of cognitive reserve. However, the attenuation was minimal and this in itself is insufficient to support the $\mathrm{DOHaD}$ cognitive reserve hypothesis of cognitive aging in this study due to several methodological limitations: Only proxy measures of brain and cognitive reserve were explored as pathway variables; direct measurements of growth and development in childhood, peak cognitive reserve, and brain volumes in adult life and brain pathology in late life were not available in this cohort. Nevertheless, attained educational level was an independent determinant of cognitive function in late life in this cohort. This finding, the first from a lifecourse epidemiology study in a LMIC setting, is consistent with a large body of evidence from HICs synthesized in three independent systematic reviews that observe that higher attained education level as a proxy for cognitive reserve is directly related to higher scores on cognitive function and inversely with rates of cognitive impairment and dementia in late life, independent of measurements of brain volumes and pathology (Meng and D'Arcy, 2012; Chapko et al., 2018; Opdebeeck et al., 2016). The contribution of education towards cognitive reserve in this cohort will be better manifest in future follow-up studies examining cognitive decline.

It is unclear why only certain measurements of birth size, but not others, were related to cognitive abilities in late life in this and other similar studies. Lack of accuracy in measurements of birth size and not adjusting for gestational age are two plausible explanations. Gestational age, as an estimate from the date of last menstrual period, was available for only a third of the participants in this cohort and was most commonly reported by mothers while in labor. Therefore, birth weight was not adjusted for gestational age, which may have reduced the specificity of the birth weight as a measure of fetal growth. Therefore, it was impossible to differentiate the influence of smaller birth size due to intrauterine growth restriction (small for gestational age) or prematurity on cardiometabolic and cognitive outcomes in this study. This, along with lack of accuracy in measurements of head circumference and length at birth, due to the greater technical difficulty of these measurements, the absence of a standard protocol when the measurements were made and rounding off of these measurements by midwives at birth may have influenced the associations of these birth size measures with cognitive outcomes in this study. In the MYNAH cohort, nearly $50 \%$ of the cohort members had their head circumference measurements rounded off to 13 inches, and a further $30 \%$ of them had their measurements of length at birth rounded off to 21 inches). It is also plausible that birth weight may have been more affected by intrauterine growth restriction than leg length and head circumference. The lack of associations of head circumference with cognition may also be due to the fetal brain sparing adaptation effect; relatively little restriction of fetal brain development is known to occur as result intrauterine growth restriction, when compared to other organ systems like musculoskeletal growth (Miller et al., 2016).

Cognitive decline is thought to begin as early as forty years of age (Singh-Manouex et al., 2012), and it is likely that we may have measured decline rather than peak cognitive ability in this study. The battery of cognitive tests employed in this study, despite its culture and education fair properties, did not examine several domains of cognitive function in detail, such as executive function, frontal lobe function, and non-verbal memory, which have been related to size at birth in several studies (Skogen et al., 2013; Raikkonen et al., 2013; Muller et al., 2014). 
Studies from HICs indicate that higher BMI in mid-life and lower BMI in late life is associated with higher risk of cognitive decline and dementia in late life (Kivimaki et al., 2018). However, in this study, higher BMI in both mid-life and late life was directly related to higher composite cognitive score in late life. The most likely reasons for this observation in the complex confounding structure of SEP and education on this relationship, higher levels of SEP, and attained education were related to higher $\mathrm{BMI}$ and cognitive function in late life in this cohort.

\section{Strengths}

In addition to measurements of size at birth, indicators of SEP in early life and in mid-life were available for the MYNAH participants, which is unusual for cohorts of this age. Furthermore, unlike previous studies (reviewed in detail in Krishna et al., 2019) which were mostly limited to reporting associations of birth size with cognitive function, this study was specifically designed to explore the different $\mathrm{DOHaD}$ pathways of cognitive aging. Another strength of our study is the completeness of the data available for analyses: Birth weight (exposure) and cognition (outcomes) were available for all $(n=721)$, and diagnosis of cardiometabolic disorders was established in almost all of them $(n=716)$. Asessments for cognitive function and mental health were conducted using validated and culturally adapted instruments. Same protocol for physical health assessments and investigations to diagnose cardiometabolic disorders was employed in the earlier and current study.

\section{Limitations}

This cohort was not a true "population" sample. It was based on all births within a single major hospital during selected years, who were still alive, lived locally, and provided sufficient information to be able to match them to their birth records with certainty. The representativeness analyses indicated that those who had survived since the original study and examined in this study were not only heavier at birth, but also had better education levels as adults and better cardiometabolic health and socioeconomic position in mid-life and late life. It is difficult to say how these various factors have influenced the representativeness of the traced cohort sample. Losses to follow-up due to the reasons mentioned above could attenuate associations between newborn size and adult health outcomes among survivors but are unlikely to create spurious associations. In a within-cohort analysis, such bias would be introduced only if the associations between fetal growth and adult outcomes differed between those studied and not studied. As mentioned earlier, insufficient information from birth records to accurately estimate gestational age, the probable lack of accuracy in certain measurements at birth, less detailed neuropsychological assessment, and inability to employ structural equation modeling are some of the other limitations.

Despite all the limitations, to our knowledge this is the first lifecourse epidemiology study in a LMIC setting to explore $\mathrm{DOHaD}$ pathways of cognitive aging and has demonstrated the salience of growth in early life in relation to cognitive outcomes in late life. However, body size at birth is a crude proxy for prenatal developmental milieu and early living conditions that are affected by multiple factors with potential long-term neurodevelopmental consequences. An association between birth weight and late life cognition does not prove causality, and it can be argued that these associations may be a result of several other factors from early life that were not examined in this study like genetic factors, maternal stress, and adversities other than under nutrition.

\section{Conflicts of interest}

The authors declare no competing interests.

\section{Funding}

This study was supported by an Early Career Fellowship grant awarded to Dr Murali Krishna by Welcome DBT India Alliance.

\section{Description of authors' roles}

The study was conceptualized by MK, CHDF, $\mathrm{MV}$, and MP. KK, GVK, and VSR were involved in retracing the cohort and developing protocols for conducting analyses. MKG and SCK conducted clinical evaluation of all the participants. $\mathrm{PC}$ and $\mathrm{KN}$ were help with data management. CO, $\mathrm{CHDF}, \mathrm{MK}$, and GVK conducted the statistical analyses. GC conducted the genetic analyses. All authors have contributed to the preparation of the manuscript.

\section{Acknowledgements}

This study was supported by an Early Career Fellowship grant awarded to Dr Murali Krishna by Welcome DBT India Alliance. We are grateful to participants and their families for their cooperation and participation in this study. We acknowledge the contributions 
of the present and previous staff at Holdsworth Memorial Hospital, Mysore. They include Dr Sona Rao, Saroja A, Ramya MC, Bhavya, Praveen, Santhosh N, Somashekara R, Malathi MP, Pavithra Rani HD, Tony Gerald, Jayakumar MN, Geetha S, Annamma, Chachyamma, Tonaly Gerald Lawrence, Tony Clifford Onslow, Swaranagowri MN, Surekha, Gopal Singh, Shylaja, Harini, Shobha, Poornima, Srinivas, and Stephen Justine Manohar. We are thankful to the late Professor David JP Barker for his support in establishing the cohort and the research centre at CSI Holdsworth Memorial Hospital. We also thank other members of the MRC Lifecourse Epidemiology Unit who have contributed to the Mysore Birth Records Cohort studies including Claudia Stein, who created the cohort by tracing people born in HMH in 1993-1995.

\section{Supplementary material}

To view supplementary material for this article, please visit https://doi.org/10.1017/S1041610221001186

\section{References}

Alberti, K. G., Zimmet, P. and Shaw, J. (2006). Metabolic syndrome a new world-wide definition. A consensus statement from the International diabetes federation. Diabetes Medicine, 23, 469-480.

American Psychiatric Association. (2000). Diagnostic and Statistical Manual of Mental Disorders. Washington, DC: American Psychiatric Association.

Barker, D. J., Gluckman, P. D., Godfrey, K. M., Harding, J. E., Owens, J. A. and Robinson, J. S. (1993). Fetal nutrition and cardiovascular disease in adult life. Lancet, 341, 938-941.

Chapko, D., McCormack, R., Black, C., Staff, R., Murray, A. (2018). Life-Course determinants of cognitive reserve (CR) in cognitive aging and dementia - a systematic literature review. Aging $\mathcal{E}$ Mental Health, 22(8), 915-926. doi: 10.1080/13607863.2017.1348471.

Cook, D. G., Shaper, A. G., MacFarlane, PW. (1989). Using the WHO (Rose) angina questionnaire in cardiovascular epidemiology. International fournal of Epidemiology, 18(3), 607-613.

Copeland, J. R., Dewey, M. E. and Griffiths-Jones, H. M. (1986). A computerized psychiatric diagnostic system and case nomenclature for elderly subjects: GMS and AGECAT. Psychological Medicine, 16, 89-99.

Costa, A. J., Kale, P. L., Luiz, R. R., De Moraes, S. A., Mosley, T. H. and Szklo, M. (2011). Association between birthweight and cognitive function in middle age: the atherosclerosis risk in communities study. Annals of Epidemiology, 21, 851-856.

Epstein, H. T. and Epstein, E. B. (1978). The relationship between brain weight and head circumference from birth to age 18 years. American fournal of Physical Anthropology, 48, 471-473.
Erickson, K., Kritz-Silverstein, D., Wingard, D. L. and Barrett-Connor, E. (2010). Birth weight and cognitive performance in older women: the Rancho Bernardo study. Archives of Womens Mental Health, 13, 141-146.

Gale, C. R., Walton, S. and Martyn, C. N. (2003). Foetal and postnatal head growth and risk of cognitive decline in old age. Brain, 126, 2273-2278.

Ganguli, M. et al. (1996). Cognitive test performance in a community-based nondemented elderly sample in rural India: the Indo-U.S. cross-national dementia epidemiology study. International Psychogeriatrics, 8, 507-524.

Grove, B. J., Lim, S. J., Gale, C. R. and Shenskin, S. D. (2017). Birth weight and cognitive ability in adulthood: a systematic review and meta-analysis. Intelligence, 61 , 146-158.

Gunnel1, D. (2002). Can adult anthropometry be used as a 'biomarker' for prenatal and childhood exposures? International Fournal of Epidemiology, 31(2), 390-394.

Hall, K. S., Gao, S., Emsley, C. L., Ogunniyi, A. O., Morgan, O. and Hendrie, H. C. (2000). Community screening interview for dementia (CSI 'D'); performance in five disparate study sites. International fournal of Geriatrics Psychiatry, 15, 521-531.

Hendrie, H. C. et al. (1993). Alzheimer's disease is rare in Cree. International Psychogeriatrics, 5, 5-14.

Hyvarinen, M. et al. (2009). Impact of glucose metabolism and birth size on cognitive performance in elderly subjects. Diabetes Research and Clinical Practice, 83, 379-386.

Kivimäki, M et al. (2018). Body mass index and risk of dementia: analysis of individual-level data from 1.3 million individuals. Alzheimers Dementia, 14, 601-609.

Krishna, M. et al. (2017). Birth size, risk factors across life and cognition in late life: protocol of prospective longitudinal follow-up of the MYNAH (MYsore studies of Natal effects on Ageing and Health) cohort. BMF Open, 7, e01255210.

Krishna, M., Jones, S., Maden, M., Du, B., Mc, R., Kumaran, K., Karat, S.C., Fall, CHD. (2019). Size at birth and cognitive ability in late life: A systematic review. International fournal of Geriatric Psychiatry, 34(8), 11391169. doi: 10.1002/gps.5138.

Krishna, M., Kalyanaraman, K., Veena, S. R., Krishanveni, G. V., Karat, S. C., Cox, V., Coakley, P., Nagaraj, K., Stein, C., Paul, B, Prince, M., Osmond, C., Fall, C. H. (2015). Cohort Profile: The 1934-66 Mysore Birth Records Cohort in South India. International fournal of Epidemiology, 44(6), 1833-1841. doi: 10.1093/ije/ dyv176.

Lim, S. S. et al. (2012). A comparative risk assessment of burden of disease and injury attributable to 67 risk factors and risk factor clusters in 21 regions, 1990-2010: a systematic analysis for the Global Burden of Disease Study 2010. Lancet, 380, 2224-2260.

Macfarlane, P. W. (2000). Minnesota coding and the prevalence of ECG abnormalities. Heart, 84(6), 582-584.

Martyn, C. N., Gale, C. R., Saye, A. A. and Fall, C. (1996). Growth in utero and cognitive function in adult life: follow up study of people born between 1920 and 1943 . British Medical fournal, 312, 1393-1396.

Matthews, D. R., Hosker, J. P., Rudenski, A. S., Naylor, B. A., Treacher, B. F. and Turner, R. C. (1985). Homeostasis model assessment: insulin resistance 
and beta-cell function from fasting plasma glucose and insulin concentrations in man. Diabetologia, 28, 412-419.

Meng, X. and D'Arcy, C. (2012). Education and dementia in the context of the cognitive reserve hypothesis: a systematic review with meta-analyses and qualitative analyses. PLoS One, 7, e38268.

Miller, S. L., Huppi, P. S. and Mallard, C. (2016). The consequences of fetal growth restriction on brain structure and neurodevelopmental outcome. The fournal of Physiology, 594, 807-823.

Morris, J. C. et al. (1989). The consortium to establish a registry for Alzheimer's disease (CERAD). Part I. Clinical and neuropsychological assessment of Alzheimer's disease. Neurology, 39, 1159-1165.

Muller, M. et al. (2014). Birth size and brain function 75 years later. Pediatrics, 134, 761-770.

National Family Health Survey (NFHS). (2006). Household - Population and Housing Characteristics. New Delhi: Minstry of Health and Family Welfare, Government of India.

Opdebeeck, C., Martyr, A. and Clare, L. (2016). Cognitive reserve and cognitive function in healthy older people: a metaanalysis. Neuropsychology Development and Cognition Section B Aging and Neuropsychology and Cognition, 23, 40-60.

Prince, M. et al. (2007). The protocols for the 10/66 dementia research group population-based research programme. BMC Public Health, 7, 165.

Prince, M., Acosta, D., Chiu, H., Scazufca, M. and Varghese, M. (2003). Dementia diagnosis in developing countries: a cross-cultural validation study. Lancet, 361, 909-917.

Raikkonen, K. et al. (2013). Early life origins cognitive decline: findings in elderly men in the Helsinki birth cohort study. PLoS One, 8, e54707.
Shenkin, S. D., Deary, I. J. and Starr, J. M. (2009) Birth parameters and cognitive ability in older age: a follow-up study of people born 1921-1926. Gerontology, 55, 92-98.

Shenkin, S. D., Starr, J. M. and Deary, I. J. (2004). Birth weight and cognitive ability in childhood: a systematic review. Psychological Bulletin, 130, 989-1013.

Singh-Manouex, A et al. (2012) Timing of onset of cognitive decline: results from Whitehall II perspective cohort study. British Medical fournal, 344, d7622.

Skogen, J. C., Overland, S., Smith, A. D., Mykletun, A. and Stewart, R. (2013). The impact of early life factors on cognitive function in old age: the Hordaland health study (HUSK). BMC Psychology, 1, 16.

Stein, C. E., Fall, C. H., Kumaran, K., Osmond, C., Cox, V. and Barker, D. J. (1996). Fetal growth and coronary heart disease in South India. Lancet, 348, 1269-1273.

Sudfield, C. R. et al. (2015). Linear growth and child development in low- and middle-income countries: a metaanalysis. Pediatrics, 135, e1266-e1275.

Whalley, L. J., Dick, F. D. and McNeill, G. A. (2006). Life-course approach to the aetiology of late-onset dementias. The Lancet Neurology, 5, 87-96.

World Alzheimer Report. (2014). Dementia and Risk Reduction: An Analysis of Protective and Modifiable Factors. London, UK: Alzheimer Disease International (ADI).

World Health Organisation. (2006). Definition and Diagnosis of Diabetes Mellitus and Intermediate Hyperglycemia: Report of WHO/IDF Consultation. Geneva, Switzerland: WHO.

Zhang, Z. X. et al. (2009). Lifespan influences on mid- to late-life cognitive function in a Chinese birth cohort. Neurology, 73, 186-194. 